\title{
Fuchs Syndrome: An Uncommon Entity of Stevens-Johnson Syndrome beyond Drugs
}

\author{
Muhammed Hafis ${ }^{1}$, Kaavil V Arun ${ }^{2}$, Bhaskar Shenoy ${ }^{3}$
}

\begin{abstract}
Background: A rare case of atypical Steven-Johnson syndrome secondary to mycoplasma infection.

Case description: An eight-year-old boy presented with a history of fever and cough of 7 days with recent-onset redness and purulent discharge of eyes with multiple oral ulcers for the past 3 days. He was febrile, toxic looking with bilateral conjunctival congestion, multiple painful ulcers in the mouth, and severe mucositis. Because of cefpodoxime ingestion before the onset of ulcers and redness of eyes, possibility of StevenJohnson syndrome considered and history of respiratory infection in the absence of skin involvement, atypical Steven-Johnson syndrome secondary to mycoplasma infection (Fuchs syndrome) kept in mind. mycoplasma serology was sent which was reported as positive. Other routine investigations and chest $\mathrm{X}$-ray were normal.

Intervention: He was started on IV pulse methylprednisolone for 7 days. He becomes afebrile after the first dose of methylprednisolone. Eye lubricants and topical anti-inflammatory agents continued. A significant reduction in mucositis observed. Later oral corticosteroid continued and tapered and stopped over 2 weeks.
\end{abstract}

Message: Severe mucositis with the background of respiratory infection, Fuchs syndrome should be considered as a differential.

Keywords: Fuchs syndrome, Mycoplasma, Steven-Johnson syndrome.

Pediatric Infectious Disease (2019): 10.5005/jp-journals-10081-1232

\section{INTRODUCTION}

Stevens-Johnson syndrome (SJS) is a mucocutaneous reaction occurring post-exposure to certain drugs and also due to infection, characterized by fever and extensive mucocutaneous involvement with necrosis and epidermolysis. An atypical variant of SJS with mucosal involvement in the absence of skin lesion known as Fuchs syndrome. Most of the cases are triggered by Mycoplasma pneumoniae (M. pneumoniae). ${ }^{1-3}$

\section{Case Description}

An 8-year-old boy presented with a history of fever and cough for the past 7 days for which he was treated with oral antipyretics and antibiotics (cefpodoxime) in a peripheral center currently presented with persistent fever spikes and recent-onset conjunctival congestion with bilateral purulent eye discharge. He also had multiple oral ulceration for the past 3 days.

On initial evaluation, he was febrile, toxic looking with bilateral conjunctival congestion with active purulent discharge, erythematous lip erosions, and multiple painful oral ulcers with severe mucositis (Fig. 1). No obvious skin or genital lesions noted. No abnormalities detected in respiratory and other systemic examinations. A drug-induced mucocutaneous reaction was kept in mind initially.

Laboratory evaluation revealed normal blood counts (total count, 6,910 $\mathrm{mm}^{3}$; N, 53.2; L, 31.4, M, 15.1; hemoglobin, 12.8, platelet count, $5.35 \mathrm{~L} / \mathrm{mm}^{3}$ ), however, C-reactive protein and erythrocyte sedimentation rate were positive ( 46 and 80 , respectively). Intravenous corticosteroids were considered. Active sepsis is ruled out by negative procalcitonin and blood culture. The patient was commenced on intravenous pulse dose methylprednisolone (30 $\mathrm{mg} / \mathrm{kg} /$ day) with antipyretics and other supportive measures.

On the next day, the patient also had episodes of cough with expectoration which was blood-stained along with mucosal

\begin{abstract}
${ }^{1-3}$ Department of Paediatrics and Paediatric Infectious Disease, Manipal Hospitals, Bengaluru, Karnataka, India

Corresponding Author: Bhaskar Shenoy, Department of Paediatrics and Paediatric Infectious Disease, Manipal Hospitals, Bengaluru, Karnataka, India, Phone: +91 9845036174, e-mail: bshenoy@gmail.com How to cite this article: Hafis M, Arun KV, Shenoy B. Fuchs Syndrome: An Uncommon Entity of Stevens-Johnson Syndrome beyond Drugs. Pediatr Inf Dis 2019;1(4):171-173.

Source of support: Nil

Conflict of interest: None
\end{abstract}

erosions. He was further evaluated with chest skiagram which was nil contributory. He became afebrile with a single dose of intravenous steroids. Possibilities of mycoplasma infection with mucositis were considered because of prior history of respiratory infection with normal chest skiagram and samples sent for serology. Intravenous pulse dose methylprednisolone was continued with other supportive care including eye lubricants and topical antiinflammatory agents. Serology reported positive for M. pneumoniae $\operatorname{lgM}$ on day 5 of admission. Since he showed significant clinical improvement without any further fever spikes over the past 5 days, treatment with intravenous methylprednisolone was continued. No macrolide antibiotic was added though it is the drug of choice for mycoplasma. He responded well with the above treatment. Intravenous steroids were changed to oral prednisolone. A significant reduction of mucositis was observed at the time of discharge. Oral prednisolone was tapered and stopped over the next 2 weeks. He completely recovered at 2 weeks of follow-up.

\section{Discussion}

Stevens-Johnson syndrome with mucosal involvement in the absence of skin lesion is described as Fuchs syndrome. It is characterized by the involvement of two or more mucosal 


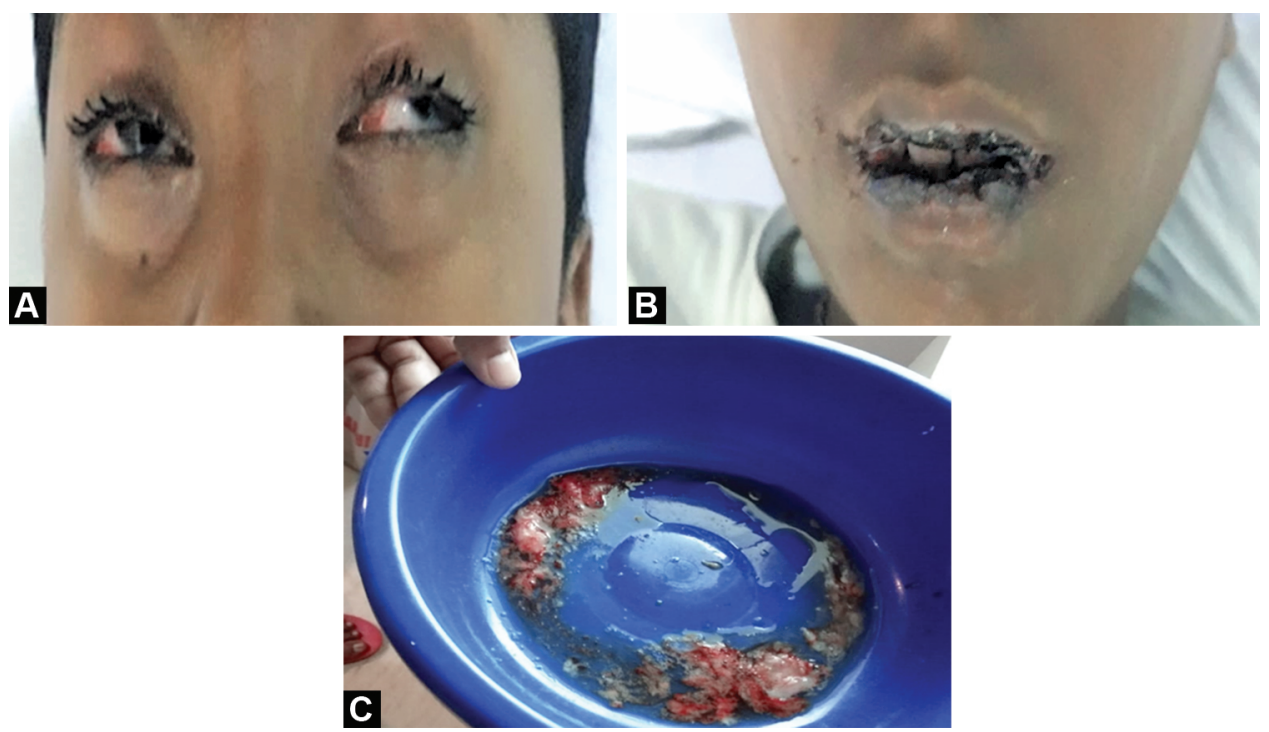

Figs 1 A to C: (A) Bilateral conjunctival congestion; (B and C) Severe mucositis involving both lips with mucosal shredding

surfaces without typical skin involvement such as purpuric macules, targetoid lesions, and full-thickness epidermal necrosis. It commonly affects the oral mucosa; however, conjunctival (67\%) and genital (75\%) involvement is also common. ${ }^{4,5}$ A higher incidence is seen in children and adolescents. ${ }^{1,4,5}$ Most cases are triggered by infections with $M$. pneumoniae and Herpes simplex where $M$. pneumoniae being the most common agent. ${ }^{3}$ Various terminologies like atypical SJS, incomplete SJS, or erythema multiforme major are also used for the same condition. Fuchs syndrome should be considered in a patient with severe mucositis without skin involvement, following a respiratory illness.

The exact pathogenesis is unknown but immunological response to the infectious agent causing generalized apoptosis of keratinocytes by T-lymphocytes and proteins like granulysin and Fas-ligand has been postulated. ${ }^{3}$

There is no single diagnostic test for Fuchs syndrome. Clinical suspicion with supportive evidence makes the diagnosis. M. pneumoniae is diagnosed by serology or polymerase chain reaction (PCR) of a throat swab. In the resource-limiting settings where serology and PCR are not available, a simple cold agglutination test can be done to increase the suspicion of infectious cause (most common M. pneumoniae) of SJS/toxic epidermal necrolysis overlap even though it is not very sensitive. ${ }^{5}$ A chest skiagram may or may not be helpful. In total, 5 to $10 \%$ of $M$. pneumoniae can have normal chest X-ray. ${ }^{6}$

There are no clear guidelines recommended for the treatment of Fuchs syndrome. ${ }^{7}$ The comprehensive studies on Fuchs syndrome are not available till date. In a confirmed case of Fuchs syndrome, macrolide antibiotics (azithromycin/clarithromycin) with other supportive management are satisfactory. Azithromycin is not considered to be a common etiologic agent for SJS; hence, it is a safe choice in the majority of patients with mycoplasma induced Fuchs syndrome. ${ }^{8,9}$

In a doubtful case where mycoplasma is not confirmed initially, Systemic corticosteroids should be considered as the first-line therapy depending on availability and affordability. Pulse therapy of methylprednisolone ( $30 \mathrm{mg} / \mathrm{kg} /$ dose), high-dose oral prednisolone ( $2 \mathrm{mg} / \mathrm{kg} / \mathrm{day}$ ), and intravenous/intramuscular dexamethasone (8-16 mg/day) are the available options, and it should be continued till the disease activity ceases and taper and stop over a total duration of 7-10 days. Other drugs like cyclosporine (3-5 mg/kg/day for 10-14 days) or low-dose intravenous immunoglobulin (0.2-0.5 $\mathrm{mg} / \mathrm{kg}$ for 24-48 hours) are also considered in a case when there is a relative contraindication to corticosteroids. ${ }^{2}$

Good supportive care is mandatory for satisfactory outcomes, especially infection control and management of hydration and dyselectrolytemia. Pain management nutritional support and care of mucous membranes are also essential. An excellent prognosis is expected with proper care and timely management. ${ }^{3,4,7}$

\section{Conclusion}

Consider Fuchs syndrome as a differential in a patient with a mucocutaneous reaction without skin involvement if there is a prior history of respiratory illness. Cases of Fuchs syndrome are probably underdiagnosed especially in pediatric practice due to lack of clinical suspicion. Lack of agreed evidence-based treatment guidelines demands further comprehensive studies on Fuchs syndrome. Suspicion beyond drug may point toward the diagnosis of Fuchs syndrome due to mycoplasma and other uncommon agents.

\section{References}

1. Mangal S, Narang T, Saikia UN, et al. Fuchs syndrome or erythema multiforme major, uncommon or underdiagnosed? Indian J Dermato Venereol Leprol [serial online] 2015;81(4):403-405. DOI: 10.4103/03786323.158640.

2. Gupta L, Martin A, Agarwal N, et al. Guidelines for the management of Stevens-Johnson syndrome/toxic epidermal necrolysis: An Indian perspective. Indian J Dermatol Venereol Leprol 2016;82(6):603-625. DOI: 10.4103/0378-6323.191134.

3. Sah R, Neupane S, Khadka S, et al. A Case Study of Stevens-Johnson Syndrome-Toxic Epidermal Necrolysis (SJS-TEN) Overlap in Mycoplasma pneumoniae Associated Tracheobronchitis. Infectious Diseases 2019;2019:5471765.

4. Meyer Sauteur PM, Gansser-Kälin U, Lautenschlager S, et al. Fuchs syndrome associated with Mycoplasma pneumoniae (StevensJohnson syndrome without skin lesions. Pediatr Dermatol 2011;28(4):474-476. DOI: 10.1111/j.1525-1470.2010.01200.x.

5. Thing CY, Shahdadpuri R. Fuchs syndrome: a case of fever, mucositis, and conjunctivitis. BMJ Case Reports 2018;2018:bcr2017223321. DOI: 10.1136/bcr-2017-223321. 
6. Susan DJ, Janaki R, Swischuk LE. Spectrum of clinical and radiographic findings in paediatric mycoplasma pneumoniae. RadioGraphics 2001;21(1):121-131. DOI: 10.1148/radiographics.21.1. g01ja10121.

7. Kheiri B, Alhesan NA, Madala S, et al. Mycoplasma pneumoniaeassociated Fuchs syndrome. Clin case rep 2018;6(2):434-435. DOI: 10.1002/ccr3.1350.
8. Anders UM, Taylor EJ, Kravchuk V, et al. Stevens-Johnson Syndrome without Skin Lesions: A Rare and Clinically Challenging Disease in the Urgent Setting. Emerg Med Open J 2015;1(2):22-30. DOI: 10.17140/ EMOJ-1-106.

9. Nappe TM, Garcia SL, Jacoby JL. Stevens-Johnson syndrome after treatment with azithromycin: an uncommon culprit. Am J Emerg Med 2016;34(3):676.e1-3. DOI: 10.1016/j.ajem.2015.06.039. 